\title{
Eye Torsion Elicited by Oscillating Gratings: Effects of Orientation, Wavelength and Stationary Contours
}

\author{
L. J. VAN RIJN,* J. VAN DER STEEN,* H. COLLEWIJN*
}

Received 19 March 1993; in revised form 28 June 1993

\begin{abstract}
We studied binocular cyclorotatory (torsional) eye movements in response to gratings that oscillated sinusoidally in a frontal plane. The square-wave gratings viewed by the right and left eye were presented and controlled separately to induce cycloversion and cyclovergence by oscillation in phase and out of phase. Eye movements were recorded with scleral induction coils. Stimulus oscillation frequency ranged from 0.125 to $1 \mathrm{~Hz}$ and the wavelength of the gratings ranged from 0.92 to $25.75 \mathrm{deg}$ of visual angle. Cycloversion and cyclovergence gain were, on average, comparable in magnitude and decreased with increasing oscillation frequency. There was no consistent effect of the wavelength on the magnitude of the responses. In general, responses were considerably higher to gratings that were oriented horizontally than to those oriented vertically. This anisotropy was present both in cycloversion and cyclovergence. It was enhanced in a larger sized stimulus and by presenting stationary, orthogonal contours (mimicking a "shear" movement), but it was not consistently influenced by wavelength. Cyclovergence showed a phase lag, which increased with oscillation frequency but which was independent of wavelength. In contrast, cycloversion showed a slight phase lead which was independent of both oscillation frequency and wavelength.
\end{abstract}

Eye movements Cycloversion Cyclovergence Human Coils

\section{INTRODUCTION}

Binocular cyclorotatory eye movements in response to visual stimuli can be divided into conjugate cycloversion and disjunctive cyclovergence movements, analogous to horizontal and vertical version and vergence movements. During cycloversion the eyes rotate in parallel about their optical axes; during cyclovergence they rotate in opposite directions. The most obvious potential function of cyclovergence is to promote retinal correspondence. Dynamic cycloversion has been considered (since Brecher, 1934) as a torsional optokinetic response, serving to reduce retinal slip in torsional direction. In this paper we describe some properties of cyclovergence and cycloversion induced by visual gratings.

The first goal of this study was to resolve apparent controversies between our previous results and reports from other investigators. Several authors have reported that stimuli containing horizontal contours elicit much larger cyclovergence responses than stimuli containing vertical contours (Crone \& Everhard-Halm, 1975; Howard, 1991). In a previous experiment (Van Rijn, Van der Steen \& Collewijn, 1992) we also found such a difference, but it was much smaller than reported by the

*Department of Physiology I, Faculty of Medicine, Erasmus University, Rotterdam. P.O. Box 1738, 3000 DR Rotterdam, The Netherlands. other authors, cited above. Our measurement technique and that of Howard were similar, but some differences existed between his and our experiments. The size and density of the stimulus gratings were different and, moreover, Howard used composite ("shear") stimuli, i.e. stimuli consisting of both a horizontal and a vertical grating, of which only one was oscillating. In the present study we investigated the effect of stimulus configuration on the anisotropy of cyclovergence responses. In addition we investigated whether a similar anisotropy was present for cycloversion.

To further elucidate the function and mechanism of cyclovergence and cycloversion, these response types were compared with each other under various stimulus conditions. We recently reported that at an oscillation frequency of $0.2 \mathrm{~Hz}$ the magnitude of the responses of cycloversion and cyclovergence, averaged across subjects, were about similar (Van Rijn et al., 1992). Within subjects, however, these response magnitudes were uncorrelated. Also, cyclovergence and cycloversion showed marked differences in phase lag. Both cycloversion and cyclovergence have been previously studied as a function of oscillation frequency of the stimulus [cycloversion (Cheung \& Howard, 1991); cyclovergence (Howard \& Zacher, 1991)], but because of differences in experimental conditions, the results of most prior work cannot be directly compared. We investigated, as a second goal of this study, the behaviour of cyclovergence and 
cycloversion in one single experiment and at various (low) stimulus oscillation frequencies.

We found that the predominance of the response to horizontal contours over that to vertical contours occurred in cycloversion as well as cyclovergence. The magnitude of this anisotropy was influenced both by the presence of stationary contours and by the size of the stimulus but was unaffected by stimulus wavelength or oscillation frequency.

\section{METHODS}

Subjects

Eight human subjects (one female, seven males; age range 23-55 yr) participated in the experiments after giving informed consent. Their visual acuity, measured with a Snellen letter chart while the subjects wore their normal spectacle correction, was at least 1.0 in each eye. All subjects had normal binocular vision, with stereoacuities not worse than $60 \mathrm{sec}$ arc in the TNO test for stereopsis (Medical Workshop, Groningen, The Netherlands).

\section{Recording of eye positions}

The experimental design was identical to that described in Van Rijn et al. (1992). Subjects were seated at the centre of the magnetic field of a scleral-coil eyeposition recording system. Their heads were supported by a chin rest. Visual stimuli were backprojected on a translucent, frontoparallel screen at $145 \mathrm{~cm}$ distance. The images were presented dichoptically. Separation of the images for the left and right eye was achieved by red and green filters mounted on the slide projectors and on goggles. The slides could be oscillated about their optical axes by galvanometers (General Scanning, Watertown, Mass.), either in phase (cycloversion) or out of phase (cyclovergence). Eye movements were measured in three dimensions (horizontal, vertical and torsion) by scleral induction coils of the combination type (Skalar, Delft, The Netherlands). Eye-position signals were low-pass filtered at a cut-off frequency of $62.5 \mathrm{~Hz}$, sampled at $125 \mathrm{~Hz}$ and stored by a minicomputer for off-line analysis, together with the position signals of the galvanometers. All data were corrected for coil misalignment by a matrix transformation, in order to obtain eye positions relative to an earth-fixed (Fick type) coordinate system and free of cross-coupling artifacts (Ferman, Collewijn, Jansen \& Van den Berg, 1987). Cyclovergence and cycloversion were calculated for each sample as the difference between and the mean of the torsional positions of the left and right eyes. Saccades and blinkrelated eye movements were removed from the cyclovergence and cycloversion tracings by a computer routine. This routine removed all sections of the tracings in which eye velocity exceeded $15 \mathrm{deg} / \mathrm{sec}$. The correctness of this procedure was verified in all measurements by visual inspoction. After removal of trends and biasses, eye and stimulus signals were fast Fourier transformed and gain and phase were calculated from the com- ponents. Gain was computed as eye cyclovergence: stimulus cyclovergence and eye cycloversion/stimulus cycloversion in cyclovergence and cycloversion trials, respectively. Of all the raw data, a fraction of $5.6 \%$ was rejected because of excessive blinks. If in any trial the gain was below 0.05 , we did not calculate the phase lag to avoid unreliable results at low energy levels. For this reason, $20.0 \%$ of the (non-rejected) trials from the first experiment (see further) and $9.8 \%$ of those from the second experiment were excluded from phase calculations.

\section{Visual stimuli}

Gratings were oscillated in cyclovergence or cycloversion and presented to the subject in a number of configurations (Fig. 1). The order of presentation of these configurations was pseudo-random.

In Experiment $I$, the following configurations were tested: (1) the orientation of the oscillating grating was either horizontal or vertical; (2) the frequency of oscillation was $0.125,0.25,0.5$ or $1 \mathrm{~Hz}$; (3) in half of the measurements a secondary, stationary grating, oriented orthogonally to the average orientation of the moving grating was superimposed, to mimic a shear-stimulus as used by Howard (1991). All possible combinations of these conditions were presented to each subject, both for cyclovergence and for cycloversion. The wavelength of the grating, i.e. the width of one black plus one white bar, was $3.00 \mathrm{deg}$ of visual angle. Seven subjects participated in this experiment.

In Experiment $I I$, we elaborated on the role of wavelength. In this experiment we used gratings with wavelengths of $0.92,2.03,4.73,8.65,16.55$ or $25.75 \mathrm{deg}$ of visual angle. All gratings were presented horizontally as well as vertically. The oscillation frequency was always $0.25 \mathrm{~Hz}$ and no stationary gratings were superimposed. This experiment was performed on four subjects. Each condition was measured twice within each subject.

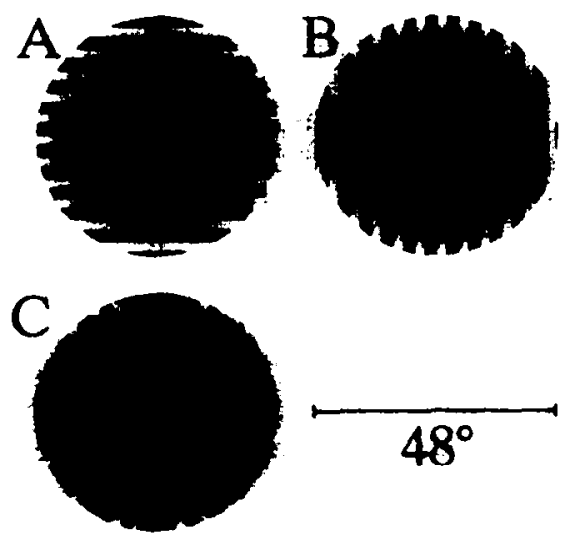

FIGURE 1. The stimulus patterns that were used in these experiments were isolated horizontal (A) or vertical (B) gratings or a combination of both (C). Of these combined ("composite") gratings either the horizontal or the vertical component was oscillated. In Expt I all stimulus types were used. In Expt II only isolated gratings were used. of which the wavelength was varied. (Wavelength was the width of one black + one white bar in deg of visual angle.) 
In both experiments, the shape of the stimulus was circular with a diameter of $48 \mathrm{deg}$ of visual angle. The amplitude of the stimulus cyclovergence or cycloversion was $4 \mathrm{deg}$ peak-to-peak. Hence in the cyclovergence measurements the amplitude of the stimulus oscillation to each eye was half of that in the cycloversion measurements.

\section{Statistical procedures}

Data were analyzed with univariate analysis of variance, linear regression, paired or grouped $t$-tests or a Student-Newman-Keuls test, using the SPSS-PC statistical software. Reported $P$-values of $t$-tests were calculated assuming two-sided alternative hypotheses. In order to normalize data distributions, all gain values were log-transformed prior to statistical testing.

\section{RESULTS}

\section{Experiment I}

Effects of oscillation frequency, moving-grating orientation and presence of a stationary grating

All stimuli elicited distinct sinusoidal cyclorotation responses under all conditions. Horizontal moving gratings always elicited higher responses than vertical moving gratings and, in general, the addition of a stationary grating inhibited the responses. Gain de- creased with increasing oscillation frequency. Figure 2 shows some examples of recordings obtained from cyclovergence and cycloversion measurements, illustrating the effect of grating orientation.

Figure 3 shows an overall gain and phase diagram for the first experiment. Figure 3(A) shows gain for cyclovergence (left) and cycloversion (right).

We compared the response magnitudes (gain) in an analysis of variance. Both for cyclovergence and cycloversion, the effects of the orientation of the moving grating, the presence of an additional stationary grating, and the oscillation frequency were all significant. All interactions between these factors were not significant, except for the interaction between the orientation of the moving grating and the presence of a stationary grating. In order to further examine the effects of these latter two factors, we compared the four groups formed by the different combinations of the factors "orientation of the moving grating" and "presence of a stationary grating", in a Student-Newman-Keuls test (significance level 0.05 ). The differences between the responses to the moving horizontal grating and to the moving vertical grating were significant, both in the presence and in absence of a stationary grating. A stationary grating inhibited the response significantly only when a stationary horizontal grating was added to an oscillating vertical grating; the addition of a stationary vertical grating to an oscillating horizontal grating did not significantly

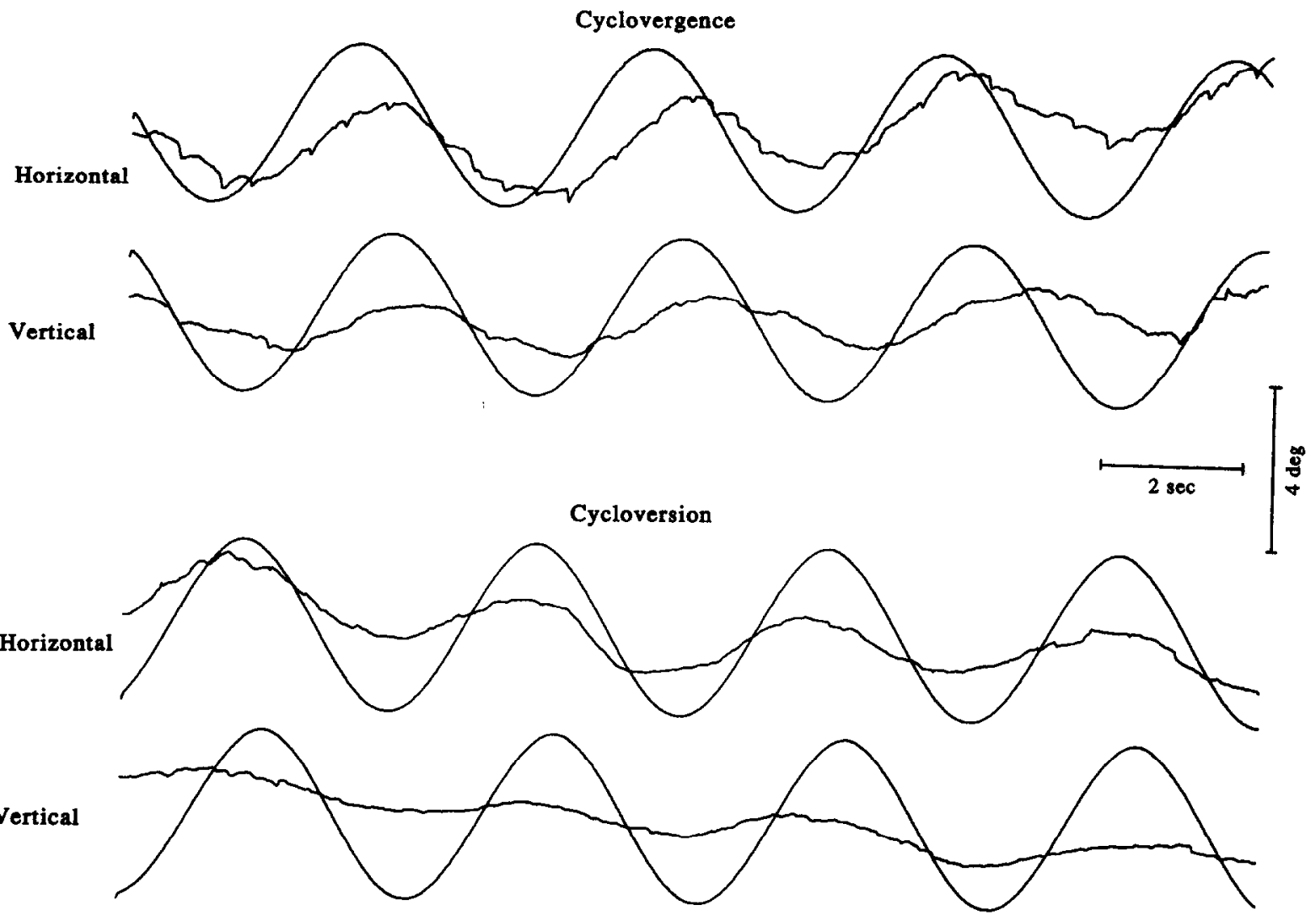

FIGURE 2. Examples of tracings obtained in Expt I, demonstrating the effect of grating orientation. Isolated gratings, either horizontal or vertical, were presented at an oscillation frequency of $0.25 \mathrm{~Hz}$. The regular sine wave reflects the (cyclovergence or cycloversion) movement of the stimulus. Gain values of cyclovergence were 0.50 and 0.30 ; gain values of cycloversion were 0.27 and 0.16 , for horizontal and vertical gratings, respectively. 

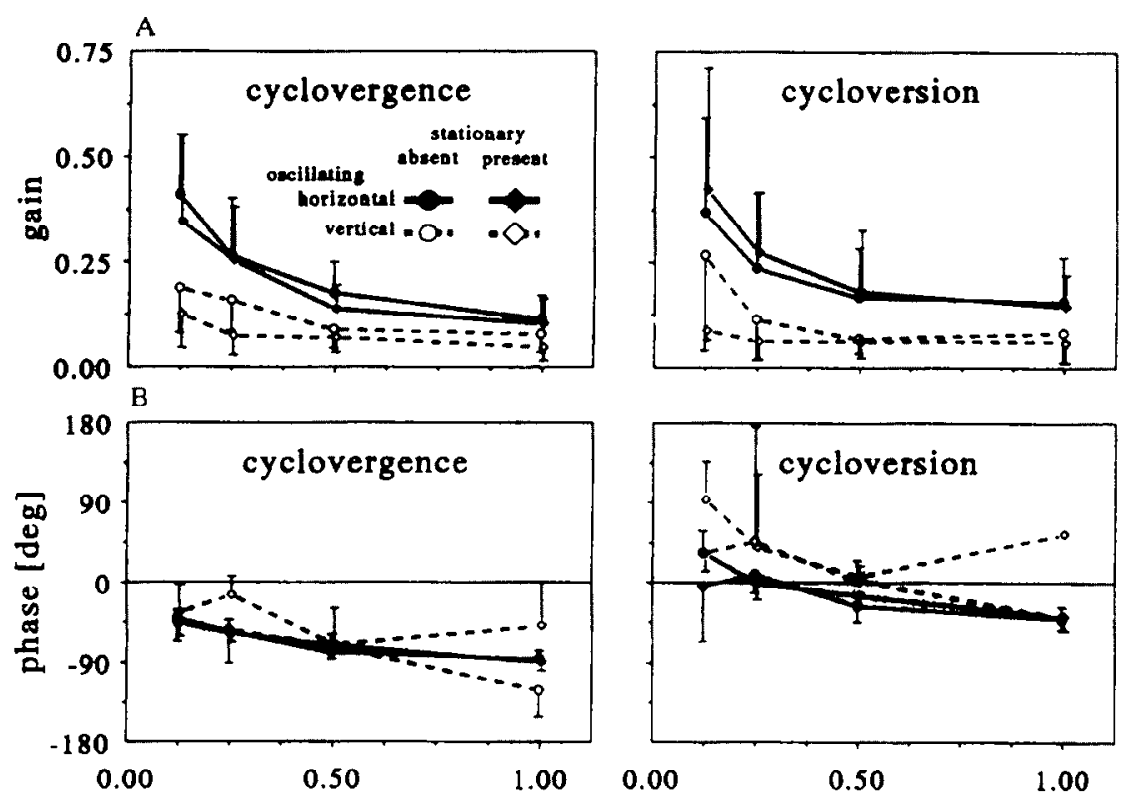

frequency $[\mathrm{Hz}]$

\begin{abstract}
FIGURE 3. Gain and phase of cyclovergence and cycloversion as function of oscillation frequency of the stimulus. Plotted are the mean \pm SD of all seven subjects. The gain of both cyclovergence and cycloversion (A) was higher in response to horizontal gratings than in response to vertical gratings. Both response types were inbibited when a horizontal stationary grating was added to an oscillating vertical grating, but the inhibition of the response to an oscillating horizontal grating by the addition of a stationary vertical grating was not significant. Docrease in gain with increasing stimulus oscillation frequency was similar for both movement types. The phases of cyclovergence and cycloversion (B) were clearly different. In general, cyclovergence displayed a phase lag which depended on the oscillation frequency of the stimulus. Cycloversion displayed a slight phase lead which was unrelated to oscillation frequency. Markers have been plotted slightly left and right to the actual oscillation frequencies to enhance readability of the figure.
\end{abstract}

inhibit the response. Thus, oscillating horizontal gratings elicited higher responses than oscillating vertical gratings; also, stationary horizontal gratings were more effective in inhibiting responses. This applied to both cyclovergence and cycloversion.

Figure 3(B) shows the phase lags of the responses in Expt I. Cyclovergence, in general, showed a phase lag (overall mean $\pm \mathrm{SD}:-62.6 \pm 31.0 \mathrm{deg}$ ). For three stimulus conditions (isolated horizontal grating, isolated vertical grating, horizontal moving + vertical stationary grating) the relations between phase lag and stimulus frequency were similar. For these conditions phase lag increased with increasing frequency, until a plateau was reached at about $-90 \mathrm{deg}$. The fourth stimulus (vertical moving + horizontal stationary) displayed large variability. This may well be due to a low gain in response to this stimulus, which cause phase measurements to be less reliable.

For cycloversion, phase was generally close to zero, but variability was large (overall mean $\pm \mathrm{SD}$; $7.2 \pm 52.5 \mathrm{deg}$ ), despite the fact that the overall gain was of similar magnitude as that of cyclovergence (see Discussion).

\section{Experiment II}

\section{Effects of wavelength of grating}

Figure 4 shows the results of Expt II, in which the wavelength of the grating was varied. No stationary gratings were added in this experiment. The wavelength of the stimulus pattern had no systematic effect on either cyclovergence or cycloversion. This was confirmed by linear regression; the slope of the regression line was not significantly different from zcro for either cycloversion or cyclovergence, irrespective of whether the gratings were horizontal or vertical. (All slopes were $<0.031 \mathrm{log}$ unit/deg of wavelength; all $P$-values were $>0.12$.) The rather high standard deviations in Fig. 4 were mainly due to differences between subjects. Experiment II further confirmed the difference between responses to horizontal and vertical gratings that was found in Expt I.

The phase lags of the responses in Expt II are shown in Fig. 4(B). They are in agreement with the cyclovergence-cycloversion difference found in Expt I. Average phase showed a lag for cyclovergence $(-52.1 \pm 14.5 \mathrm{deg}$ for horizontal and $-66.6 \pm 41.9 \mathrm{deg}$ for vertical gratings), while a lead was found for cycloversion $(24.0 \pm 27.2 \mathrm{deg}$ for horizontal and $29.0 \pm 40.7 \mathrm{deg}$ for vertical gratings). The difference between the phase of the responses to horizontal and vertical gratings was significant for cyclovergence $(P=0.027)$, but not for cycloversion $(P=0.306)$. There was no effect of stimulus wavelength on phase of either cyclovergence or cycloversion, either for horizontal or for vertical gratings (all $P$-values $>0.38$ ).

\section{Role of movement type}

In the analysis, up to this point, cyclovergence and cycloversion were treated separately. At this stage we 


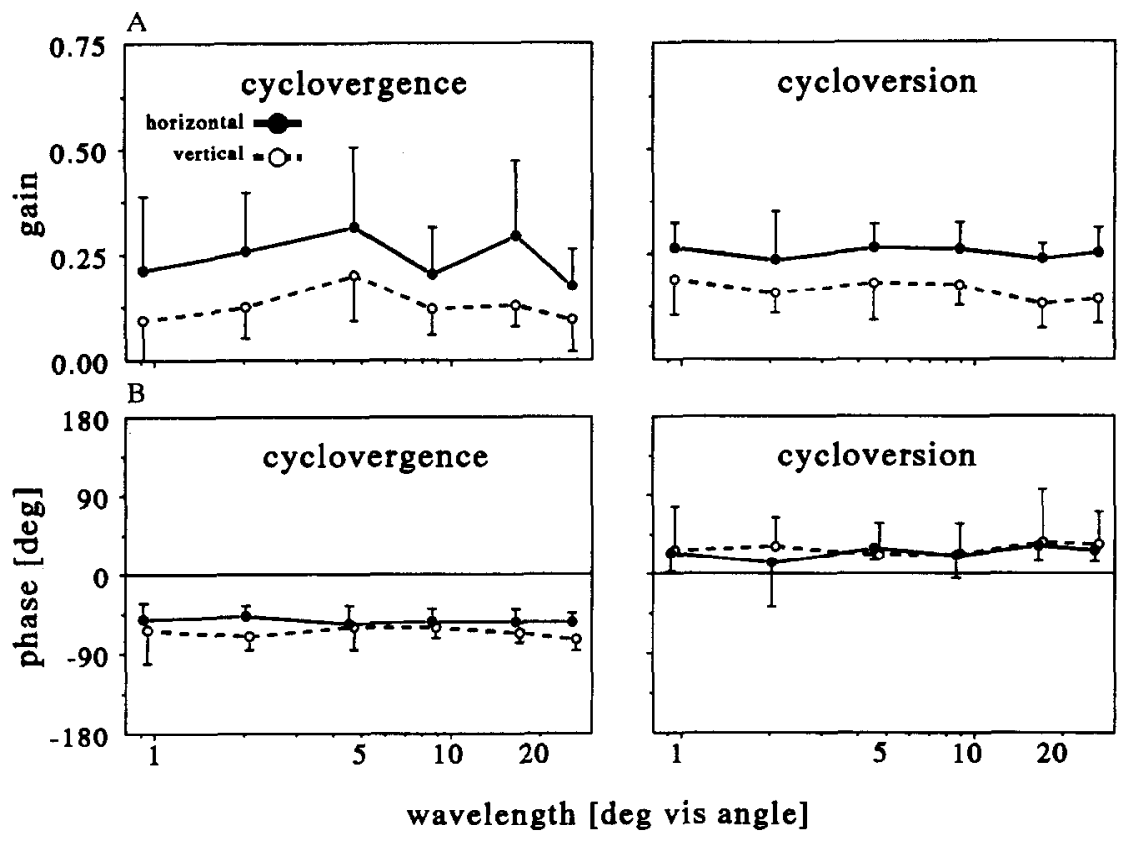

FIGURE 4. Gain and phase of cyclovergence and cycloversion as functions of the wavelength of the stimulus. Plotted are the mean \pm SD of all four subjects. The gain (A) of neither cyclovergence nor cycloversion displayed a systematic dependence on stimulus wavelength. The variability was larger for cyclovergence than cycloversion, but overall response magnitudes were similar. The phases are shown in (B). Cyclovergence displayed a phase lag; cycloversion showed a phase lead. Both were independent of the wavelength. Markers have been plotted slightly left and right of the actual oscillation frequencies to enhance readability of the figure.

compare overall response magnitudes of cyclovergence and cycloversion.

In the first experiment gain values of cyclovergence and cycloversion were not significantly different (paired $t$-test: $P=0.693$ ). In the second experiment, gain values were slightly lower for cyclovergence than cycloversion, particularly so for the vertical grating. The differences were, however, small (gain for horizontal gratings, $0.247 \pm 0.153$ and $0.253 \pm 0.067$; for vertical gratings, $0.127 \pm 0.082$ and $0.163 \pm 0.066$ for cyclovergence and cycloversion, respectively).

In both Expts I and II analysis of variance showed no interaction between the effects of movement type (cyclovergence or cycloversion) and either oscillation frequency (Expt I, $P=0.360$ ) or presence of stationary contours (Expt II, $P=0.610$ ). Hence the decrease in gain due to the addition of stationary contours was similar for cyclovergence and cycloversion. The interaction between movement type and subject was significant in both experiments $(P=0.003)$. This means that cyclovergence-cycloversion differences were subject dependent. It confirms our earlier observations (Van Rijn et al., 1992). There was no significant interaction between movement type and oscillation frequency.

\section{DISCUSSION}

In a previous investigation (Van Rijn et al., 1992) we found that horizontal contours were only slightly more effective than vertical contours in eliciting cyclovergence. That result seemed at odds with the findings of Howard (1991) and Crone and Everhard-Halm (1975), who found that horizontal gratings were $4-5$ times and 2-3 times, respectively, more effective than vertical gratings. In this study, we investigated a number of stimulus variables that may be at the basis of this discrepancy: (1) the presence or absence of stationary contours, resulting in shear or real rotational stimuli; (2) the oscillation frequency; and (3) the wavelength of the grating. We will add the size of the stimulus as a fourth variable, by comparing the present results to those from our previous experiment.

In addition, we compared horizontal contours to vertical contours as stimuli for cyclorotation for both cyclovergence and cycloversion.

\section{Rotation vs shear}

We found that the response to an oscillating vertical grating was inhibited by a stationary horizontal grating. In contrast, the inhibitory effect of a vertical stationary grating on the response to an oscillating horizontal grating was not significant. This difference in inhibition is in agreement with the difference between the responses to both grating orientations: a stimulus eliciting a large response, when moving, should be expected to also exert a large inhibitory effect, when stationary. As a consequence, stimuli that are composed of a horizontal and vertical grating display a large difference between responses elicited by oscillations of the horizontal and vertical components. The responses to vertical shear are much larger than the responses to horizontal shear. This difference is larger than the difference between the responses elicited by horizontal and vertical gratings alone. Our composite stimulus closely resembled the 
shear stimulus that was used by Howard (1991). Since in our previous study we only used purely horizontal or vertical gratings, this accounts for (part of) the discrepancy. It does not explain the discrepancy between our result and those of Crone and Everhard-Halm (1975), who also did not add stationary gratings. However, they performed objective measurements in one subject only.

In our reasoning we implicitly assumed that the cyclovergence and cycloversion systems respond to the separate oriented elements of the stimulus. A different approach would be to assume that these systems respond to the stimulus as a whole. As stated above, our composite stimulus has the character of a shear stimulus. Shear can be decomposed in distortion and rotation (e.g. Feynman, Leighton \& Sands, 1964). The rotation component of a shear stimulus equals half the angle of shear. Therefore, if the oculomotor system were to respond to this rotation component of the shear stimulus as a whole, our effective stimulus amplitude would be only half as large as stated in the Methods. The gain would therefore be twice as high. This interpretation would make the responses to shear stimuli much larger than the responses to horizontal or vertical gratings alone, and therefore this mechanism is unlikely. Another argument against this hypothesis is that the rotation component of the horizontal shear stimulus (i.e. vertical grating oscillating + horizontal grating stationary) is as high as that of the vertical shear stimulus, while the responses to these two "shear types" were very different. Thus, we conclude that the differently oriented components of the stimuli were initially processed by separate channels, being relayed with a different gain to the oculomotor system.

\section{Oscillation frequency}

We found that the difference between responses to horizontal and vertical contours did not depend on the oscillation frequency: there was no interaction between oscillation frequency and grating orientation, either for cycloversion or cyclovergence. For cyclovergence, this lack of interaction is in agreement with the figure presented in Howard (1991).

\section{Wavelength}

Another possible origin of the discrepancy with previous authors could have been the wavelength of the stimulus grating. From the figures in Crone and Everhard-Halm (1975) and in Howard (1991), it can be inferred that in those studies the predominant wavelengths were approx. 5 and $3 \mathrm{deg}$ of visual angle. In the first experiment of the present study it was $2.5 \mathrm{deg}$ of visual angle. In our previous study however, wavelength was smaller: $1.7 \mathrm{deg}$. This possible source of the discrepancy is made unlikely by our findings from the second experiment of this study. It appears that there is no systematic dependence of cyclovergence or cycloversion on stimulus wavelength, either for horizontal or for vertical gratings.

\section{Stimulus size}

Stimulus size may also lie at the basis of the discrepancy. Howard (1991) used a stimulus that subtended about $80 \mathrm{deg}$ of visual angle; in our previous experiment the stimulus size was about $28 \mathrm{deg}$. In the present experiment, the stimulus size was intermediate $(48 \mathrm{deg})$. These latter two experiments cannot be compared directly because response magnitudes also depended on other factors, which were varied in the present and previous experiments (present experiment, oscillation frequency; previous experiment, oscillation amplitude). To circumvent this problem we calculated the ratio of responses to horizontal gratings to those to vertical gratings. With analysis of variance we could not demonstrate that this ratio depended either on oscillation frequency or on oscillation amplitude. Also, there was no dependence on wavelength. Therefore, this ratio could be compared across these experiments. Table 1 lists the ratios for the different stimulus conditions in the present experiment and for our previous experiment.

TABLE 1. Mean \pm SD of ratios of responses to horizontal grating/responses to vertical grating in the different experiments

\begin{tabular}{|c|c|c|c|c|}
\hline Experiment & Type of experiment & $\begin{array}{l}\text { Cyclovergence } \\
\text { response }\end{array}$ & $\begin{array}{l}\text { Cycloversion } \\
\text { response }\end{array}$ & Size \\
\hline Ia & $\begin{array}{l}\text { Variation of oscillation } \\
\text { frequency, stationary } \\
\text { grating absent }\end{array}$ & $2.28 \pm 1.80(n=56)$ & $3.00 \pm 3.30(n=56)$ & $48 \mathrm{deg}$ \\
\hline $\mathrm{Ib}$ & $\begin{array}{l}\text { Variation of oscillation } \\
\text { frequency, stationary } \\
\text { grating present }\end{array}$ & $-3.54 \pm 3.19(n=56)$ & $5.23 \pm 4.62(n=56)$ & $48 \mathrm{deg}$ \\
\hline II & $\begin{array}{l}\text { Variation of wavelength } \\
\text { of grating, stationary } \\
\text { grating absent }\end{array}$ & $2.49 \pm 1.78(n=96)$ & $1.77 \pm 0.98(n=96)$ & $48 \mathrm{deg}$ \\
\hline IIl Van Rijn et al. (1992) & $\begin{array}{l}\text { Variation of amplitude, } \\
\text { stationary grating absent }\end{array}$ & $1.30 \pm 0.41(n=36)$ & $1.25 \pm 0.54(n=36)$ & $28 \mathrm{deg}$ \\
\hline
\end{tabular}

"Stationary present" indicates that superimposed on the oscillating horizontal grating (numerator) there was a stationary vertical grating and superimposed on the vertical grating (denominator) there was a stationary horizontal one. Data are pooled over subjects, grating orientations (i.e. horizontal or vertical) and over the factor that was varied in the considered experiment (see second column). Comparing rows Ia and II with row III by grouped $t$-test revealed that differences were significant, both for cycloversion and cyclovergence (all four $P$-values $<0.013$ ). The differences between row Ia and II were not significant. 
Ratios in the previous experiment, with its smaller stimulus size, were significantly lower than all ratios in the present experiments (grouped $t$-test: all $P$-values $<0.013$ ). Since measurement techniques, data analysis and even most of the subjects were identical in these two studies, this strongly suggests that for large stimuli the predominance of horizontal over vertical contours is stronger than for small stimuli. Howard, using a large stimulus found a ratio of about 4.5 (as estimated from his figure) and we conclude that the difference in stimulus size may explain (part of) the discrepancy.

We did not use this response ratio as a primary parameter in the analysis because the scatter in response ratios was larger than in response magnitudes, particularly when responses were low.

\section{Horizontal vs vertical contours in cycloversion}

The present experiments demonstrate that the predominance of the effect of horizontal stimulus contours is present in cycloversion as well. For cyclovergence the "explanation" for this phenomenon has been teleologic: cyclodisparities of vertical contours may be caused by slant of the observed object and do, therefore, not require compensation by eye-cyclovergence, while cyclodisparities of horizontal contours always indicate cyclovergence errors and therefore do need correction (Crone \& Everhard-Halm, 1975). This explanation is plausible indeed. However, the presence of the same phenomenon in cycloversion cannot be fitted into this hypothesis since cycloversion stimuli do not contain disparities. One may think of a teleologic explanation that is slightly different: in an outdoor setting, the most prominent horizontal contour is the horizon. This is a reliable reference for calibrating one's cycloversion state, much more so than vertical (or oblique) contours. If, then, the cycloversion mechanism would "try" to stabilize the eyes relative to the outside world, horizontal contours would be the best candidates for reference marks.

An alternative explanation for the predominance of horizontal contours in cycloversion is that the same neurophysiological mechanism mediates both cycloversion and cyclovergence. Differences and similarities between cycloversion and cyclovergence response types are discussed below.

\section{Cyclovergence os cycloversion responses}

For all oscillation frequencies we found the cyclovergence and cycloversion responses (gain) to be in a similar range of magnitude. This confirms our earlier findings that at an oscillation frequency of $0.2 \mathrm{~Hz}$ responses were largely similar. We found no dependence of either cyclovergence or cycloversion response magnitude on the wavelength of the stimulus. It must be noted though, that the variation was larger for cyclovergence than for cycloversion (Fig. 4). (The possibility of a very weak dependence of cyclovergence on the wavelength of the stimulus cannot be ruled out completely on the basis of our results.) From these results one is tempted to conclude that cycloversion and cyclovergence are essentially similar. This is supported by our finding that the predominant role of horizontal contours is similar in both movement types. Previously (Van Rijn et al., 1992) we found distinct differences between cyclovergence and cycloversion responses: within subjects the response magnitudes of cycloversion and cyclovergence did not correlate; the phases of both movement types were very much different and both movement types did show different behaviour in response to non-corresponding stimuli. All these findings are in favour of different mechanisms for cycloversion and cyclovergence. The different phases of cycloversion and cyclovergence, as well as the poor correlation between cyclovergence and cycloversion responses within subjects, were confirmed in the present results. In view of the differences and similarities that we found, it seems best to assume that cyclovergence and cycloversion are different processes, but part of their pathways may be in common.

Our results suggest that cyclovergence must be interpreted as a truly static response. This is in agreement with reports on static cyclovergence from the literature (Kertesz, 1983) and with the results and interpretation of Howard and Zacher (1991). The stimulus for cyclovergence is a disturbance of retinal correspondence of (especially) horizontal contours. In the literature, dynamic cycloversion has been interpreted as torsional optokinetic nystagmus (Brecher, 1934; Cheung \& Howard, 1991), serving to reduce retinal slip. If this would be the single function of cycloversion, one should not expect the presence of any static cycloversion response. However, static cycloversion (i.e. cycloversion in response to a static tilted visual stimulus) has been found by different investigators (Crone, 1975; Goodenough, Sigman, Oltman, Rosso \& Metz, 1979), although its magnitude was small. Its interpretation has been in terms of a visual analogue of the otolith response (Crone, 1975).

The apparent difference between the nature of dynamic and static cycloversion disappears if one assumes that cycloversion is a truly static response as well, just as cyclovergence. The difference is that the stimulus for cyclovergence is a disturbance of retinal correspondence of (especially) horizontal contours, whereas for cycloversion, as we pointed out above, it may be the alignment of the horizontal ocular meridian with the real world horizon. This hypothesis would also explain our finding that the gain steadily increases with decreasing stimulus oscillation frequency. Strong support for this hypothesis would come from an experiment demonstrating a continuum between static and dynamic cycloversion responses. However, it is difficult to measure at still lower oscillation frequencies than we did because of the presence of a slow, spontaneous drift on cycloversion (Van Rijn \& Van der Steen, 1992). This drift was less consistently present and of smaller magnitude in cyclovergence. This is in agreement with Enright's (1990) results, demonstrating that, within periods of fixation, the variation of cyclovergence is less than that of cycloversion. Static cycloversion responses and dynamic responses at low oscillation frequencies require cautious interpretation. 


\section{Phase relations}

Previously (Van Rijn et al., 1992) we reported that at a frequency of $0.2 \mathrm{~Hz}$, cyclovergence displays a phase lag, contrary to cycloversion. Howard and Zacher (1991) reported that the phase lag of cyclovergence increases with the frequency of movement, while Cheung and Howard (1991) found that cycloversion does not display a phase lag up to a frequency of $1 \mathrm{~Hz}$. Our present data are in agreement with these previous reports. There is one difference between our results on phase and those of Howard and Zacher: the relation between oscillation frequency and phase in their results was a curve that was convex upward. The maximum phase lag was $-100 \mathrm{deg}$. We found that the convexity was pointing downward and that there was a plateau at $-90 \mathrm{deg}$. In order to interpret their findings, Howard and Zacher referred to a non-linear model proposed by Buizza and Schmidt (1985). In this model, the phase curve has both concave and convex parts, depending on the frequency. Both our results and those of Howard and Zacher may be in agreement with this model, but the maximum phase lag would be expected to be similar.

In conclusion, the present results confirm earlier reports (Crone \& Everhard-Halm, 1975; Howard, 1991) that horizontal contours are much more effective in eliciting cyclovergence than are vertical contours. This predominance of horizontal contours appeared to be present in cycloversion as well. We showed that this predominance was enhanced by the presence of stationary contours rotated by $90 \mathrm{deg}$ with respect to the oscillating contours. The predominance was less for smaller sized stimuli, but there was no consistent effect of oscillation frequency or the wavelength of the grating that was used as stimulus.

Furthermore we found that the decrease of responses with increasing stimulus oscillation frequency was similar for cyclovergence and cycloversion and that the response magnitude of neither cyclovergence nor cycloversion depended on the wavelength of the stimulus.

\section{REFERENCES}

Brecher, F. A. (1934). Die optokinetische Auslösung von Augenrollung und rotatorischem Nystagmus. Pfiügers Archiv, 234, 13\%28. Buizza, A. \& Schmidt, R. (1985). New experimental data on the cat's optokinetic responses. Is there a need to revise previous models of the optokinetic reflex? Biological Cybernetics, 51. 301304

Cheung, B. S. K. \& Howard, I. P. (1991). Optokinetic torsion: Dynamics and relation to circularvection. Vision Research. 3/. 1327-1335.

Crone, R. A. (1975). Optically induced eye torsion-II. Optostatic and optokinetic cycloversion. Albrecht von Graefes Archiv für Klinische' und Experimentelle Ophralmologie, 196, 1-7.

Crone, R. A. \& Everhard-Halm, Y. (1975). Optically induced cye torsion-1. Fusional cyclovergence. Albrecht von Graefes Archit für Klinische und Experimentelle Ophtalmologie, 195, $231 \cdot 239$.

Enright, J. T. (1990). Stereopsis, cyclotorsional "noise" and the apparent vertical. Vision Research, 30, $1487-1497$

Ferman, L.. Collewijn. H.. Jansen, T. C. \& Van den Berg. V. (1987). Human gaze stability in the horizontal, vertical and torsional direction during voluntary head movements, evaluated with a three-dimensional scleral induction coil technique. Vision Research. $27,811-828$.

Feynman, R. P., Leighton. R. B. \& Sands, M. (1964). The Feynman lectures on physics (Vol. II. Chap. 38, p. 4). Reading, Mass.: Addison-Wesley

Goodenough, D. R., Sigman, E., Oltman, P. K., Rosso, J. \& Metz, H. (1979). Eye torsion in response to a tilted visual stimulus. Vision Research. 19. 1177-1179.

Howard, I. P. (1991). Image cyclorotation. cyclovergence and perccived slant. The Engineering Society for Advanced Mobility Land. Sea, Air and Space. Technical paper series, 911392, 1 8. (I. P. Howard, York University, Toronto, Ontario, Canada M3J 1P3.) Howard, I. P. \& Zacher, J. E. (1991). Human cyclovergence as a function of stimulus frequency and amplitude. Experimental Brain Research, 85, 445-450.

Kertesz, A. E. (1983). Vertical and cyclofusional disparity vergence. In Schor, C. M. \& Ciuffreda, K. J. (Eds), Vergence eye movements: Basic and clinical aspects (pp. 317 348). London: Butterworths.

Van Rijn, L. J. \& Van der Steen, H. (1992). Stability of the human eyes in torsional direction: Short term spontaneous torsional eye movements are conjugate. Pfiügers Archiv (Suppl.), 420, R42.

Van Rijn, L. J., Van der Steen, J. \& Collewijn, H. (1992). Visually induced cycloversion and cyclovergence. Vision Research, 32. 18751883 .

Acknowledgement - We thank an anonymous reviewer for his or her extensive comments that helped to improve the readability of this paper. 